\title{
Modelling of Elastic Gearboxes Using a Generalized Gear Contact Model
}

\author{
F.L.J. van der Linden, German Aerospace Center (DLR) \\ Münchner Straße 20, 82234 Weßling, Germany \\ franciscus.linden@dlr.de
}

\begin{abstract}
The object of this paper is to present an universal model that describes the gear contact between two gears in a planar environment. The model includes elastic effects between the gear wheels. Using this model it is possible to create arbitrary spur gear connections as well as all kinds of epicyclic gearing configurations by supplying the proper external constraints. The presented model is implementated in the Modelica language and Dymola is used for the simulations.
\end{abstract}

Keywords: Elasticity, Gearbox, Epicyclic Gearing, System Modeling

\section{Introduction}

Gear transmissions are widely used in almost all engineering applications. These range from cheap plastic consumer printers, aircraft actuators up to high precision positioning drive systems. The design of these transmissions is dependent on the application. This design process ranges from " "looking up a standard gear in a catalog and hope it will work"' up to detailed dynamic analysis using Finite Elements Methods.

At the moment gear research is mainly focused on the understanding of gearboxes. Özgüven and Houser [4] wrote a model review in 1988, Parey and Tandon [6] did the same in 2003. These works present a good overview of the work done up till that time. More recent works can be sorted into 3 groups:

1. Rigid models or simple elastic systems with only rotational degrees of freedom [7, 3]

2. Coupled torsional and transversional elastic models $[9,1,8,5]$

3. Self excited gears models; gear eccentricitiy, transmission errors and stiffness variations $[3,1$, $9,5]$
Some of these mentioned works have friction effects included. Most of the recent works include a full transversional-torsional coupled model including either detailed friction effects or self excitation. There is a clear trend on an increasing model detail and complexity.

However, all the models above, are not flexible when gearing configurations like compound planetary gears or even more exotic configurations are used. In the pre-design stage of such a gearbox, reduction ratios as well as internal vibrations are usually important. In this paper a model will be presented that can simulate arbitrary elastic gearbox configurations by relying on a planar library. This approach makes it very easy to evaluate several model configurations without a lot of design work. To keep the simulation time low, the presented model does not include any friction effects, since they are often not directly necessary in the predesign stage.

\section{Gear Forces and Equations}

In this chapter the forces and torques on the gear wheels are evaluated. Since these forces and torques differ for internal- and external toothing, these aspects are treated as separate cases.

\subsection{Force and Moment balance of external toothing}

In Figure 1 a schematic overview of two gear wheels in contact are shown. The rotation of the gear wheels are $\phi_{A}$ and $\phi_{B}$, shown by the angles to the body-fixed red and blue markers on the gear wheels.

The gear ratio is defined by:

$$
\frac{r_{A}}{r_{B}}=-i
$$

This ratio is constant for each gear angle and position. 


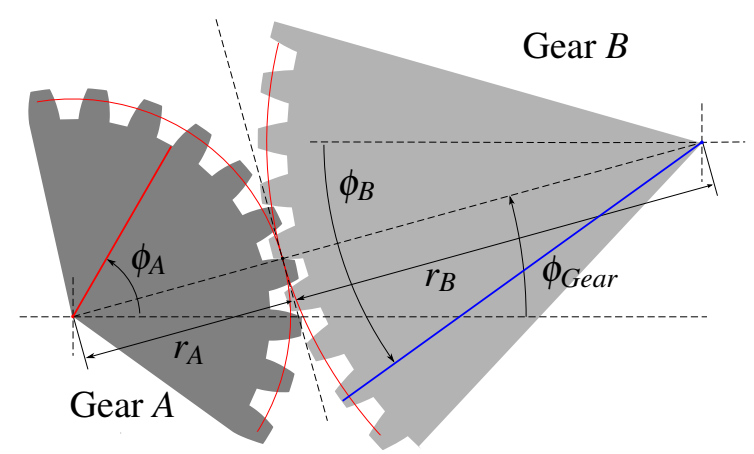

Figure 1: Schematic overview of two gearwheels in contact. The blue and red line are fixed markers on the gear wheels. In the figure $\dot{\phi}_{A}>0$ and Gear $A$ drives Gear $B$.

Figure 2 shows a free body diagram of the two gears in contact. The forces of only one contact point are displayed.

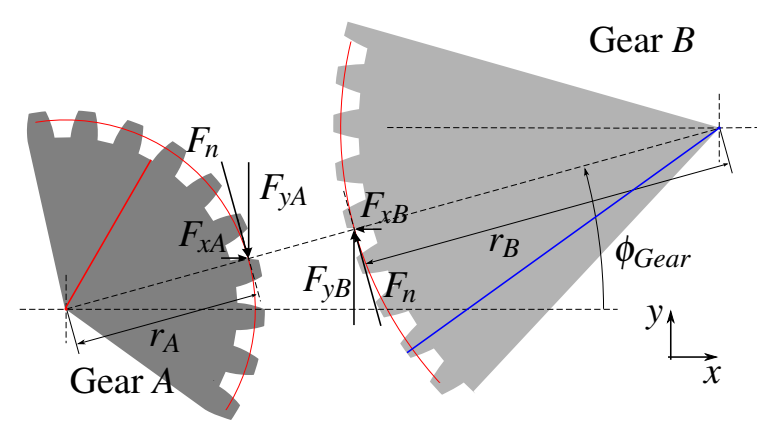

Figure 2: Free body diagram of the two gearwheels from Figure 1.

Using Figure 2, it is possible to create the torque and force balances of each gear wheel for external toothing configurations. These forces and torques are resolved in the fixed coordinate system shown in Figure 2. The use of a fixed coordinate system and gear angle $\phi_{\text {gear }}$ makes it possible to use the contact model also in more complex gear systems (e.g. all kinds of Epicyclic gearing configurations).

$$
\begin{aligned}
\tau_{A} & =F_{n} r_{A} \\
\tau_{B} & =F_{n} r_{B} \\
F_{x A} & =-\sin \left(\phi_{\text {gear }}\right) F_{n} \\
F_{y A} & =\cos \left(\phi_{\text {gear }}\right) F_{n} \\
F_{x B} & =-F_{x A} \\
F_{y B} & =-F_{y A}
\end{aligned}
$$

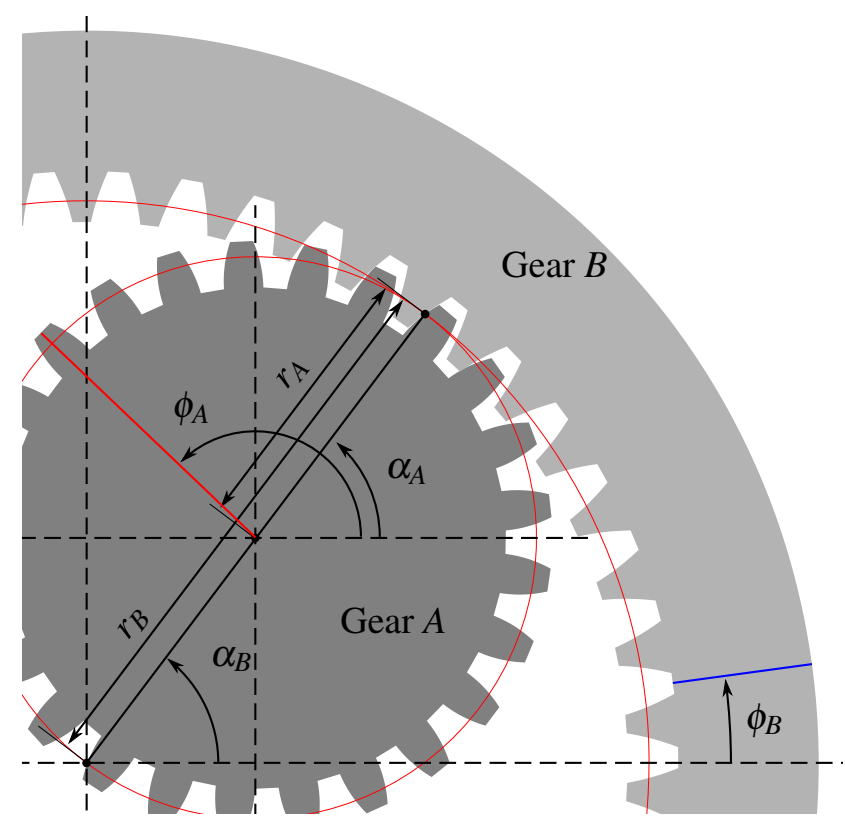

Figure 3: Schematic overview of two gearwheels in contact. The blue and red line are fixed markers on the gear wheels. In the figure $\omega_{A}>0$ and Gear $A$ drives Gear $B$.

\subsection{Force and Moment balance of internal toothing}

Just like in Section 2.1, the force and moment balance can be created by examining Figure 3 together with Figure 4:

$$
\begin{aligned}
\tau_{A} & =F_{n} r_{A} \\
\tau_{B} & =-F_{n} r_{B} \\
F_{x A} & =-\sin \left(\phi_{\text {gear }}\right) F_{n} \\
F_{y A} & =\cos \left(\phi_{\text {gear }}\right) F_{n} \\
F_{x B} & =-F_{x A} \\
F_{y B} & =-F_{y A}
\end{aligned}
$$

\section{Meshing distance}

To keep track how the gear wheels move with respect to each other, the mesh distance $x_{\text {mesh }}$ is introduced. This distance is defined as the distance the gear has traveled through the meshing point and can be calculated for both gear wheels. For the complete description of the mesh position the following assumption is postulated:

Assumption 1 The mesh contact position is on the direct connection between the center of gear $A$ and $B$ at a distance $r_{A}$ from the center of $A$ 


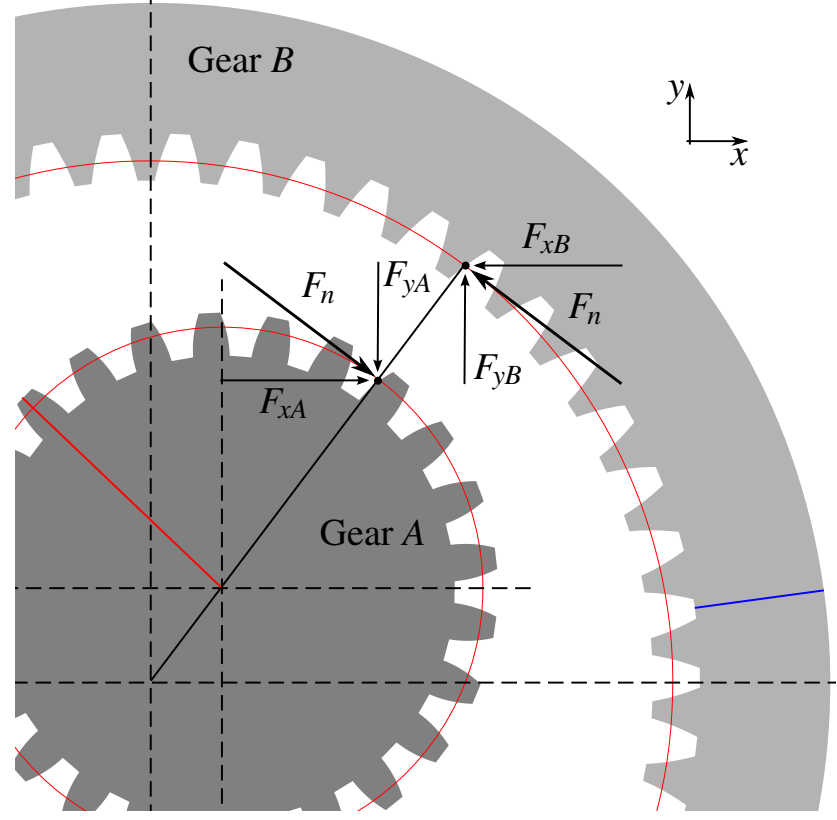

Figure 4: Free body diagram of the two gearwheels from Figure 3.

This assumption is valid for all cases in which the deformation of the tooth is small. In all engineering applications this must be the case for gearwheels under normal loading conditions.

\subsection{Mesh Distance External Toothing}

For external toothing the mesh distance can be calculated as follows using the geometry and definitions from Figure 1.

$$
\begin{aligned}
& x_{m e s h, A}=\phi_{A} r_{A}-\phi_{\text {gear }} r_{A} \\
& x_{m e s h, B}=-\phi_{B} r_{B}+\phi_{\text {gear }} r_{B}
\end{aligned}
$$

From this equation it becomes clear that the mesh distance $\left(x_{m e s h, A}\right.$ or $\left.x_{m e s h, B}\right)$ can be constant although the gear wheels are rotating. This is the case if $\phi_{A}=\phi_{\text {gear }}$ or $\phi_{B}=\phi_{\text {gear }}$. This is not only a theoretical implication; in e.g. bicycle gear hubs this is often the case.

The difference between the mesh positions is the elasticity of the gear contact:

$$
\Delta_{A B}=x_{m e s h, A}-x_{m e s h, B}
$$

Assuming the meshing position is always halfway the elastic deformation, together with using the equations 14 to 16 the mesh velocity is:

$$
v_{m e s h}=\dot{x}_{m e s h, A}-\frac{\dot{\Delta}_{A B}}{2}
$$

\subsection{Mesh Distance Internal Toothing}

The same analysis method can be applied to the internal toothing:

$$
\begin{aligned}
& x_{\text {mesh }, A}=\phi_{A} r_{A}-\phi_{\text {gear }} r_{A} \\
& x_{\text {mesh }, B}=\phi_{B} r_{B}-\phi_{\text {gear }} r_{B}
\end{aligned}
$$

The difference between the mesh positions is as mentioned above the elasticity of the gear contact:

$$
\Delta_{A B}=x_{m e s h, A}-x_{m e s h, B}
$$

Assuming the meshing position is always halfway the elastic deformation, together with using the equations 18 to 20 the mesh velocity is:

$$
v_{m e s h}=\dot{x}_{m e s h, A}-\frac{\dot{\Delta}_{A B}}{2}
$$

\section{Gear Wheel Coupling}

The gear wheels $A$ and $B$ are coupled by a springdamper combination. This yields:

$$
F_{n}=\Delta_{A B} c\left(\phi_{\text {gear }}, \phi_{A}, \phi_{B}\right)+\dot{\Delta}_{A B} d\left(\phi_{\text {gear }}, \phi_{A}, \phi_{B}\right)
$$

In this equation $c\left(\phi_{\text {gear }}, \phi_{A}, \phi_{B}\right)$ is the angle dependent spring constant and $d\left(\phi_{\text {gear }}, \phi_{A}, \phi_{B}\right)$ is the angle dependent damping constant.

\subsection{Position Dependent Stiffness}

The angle dependency can be used to simulate a non constant tooth stiffness. The total tooth stiffness is the combined stiffness of both gearwheels. Since the circumference of a gearwheel is periodic by definition, the following assumption can be postulated:

Assumption 2 The position dependent stiffness and damping of a gearwheel can be described by a Fourier decomposition.

One of the most basic forms of Assumption 2 is a single harmonic with zero phase offset that represents the tooth of the gear wheel. The stiffness over the circumference of a gearwheel can therefore be written as:

$$
\begin{aligned}
& c_{A}\left(\gamma_{A}\right)=c_{\text {const }}+c_{\Delta, A} \sin \left(2 \pi n_{\text {tooth }, A} \gamma_{A}\right) \\
& c_{B}\left(\gamma_{B}\right)=c_{\text {const }}+c_{\Delta, B} \sin \left(2 \pi n_{\text {tooth }, B} \gamma_{A}\right)
\end{aligned}
$$

In this equation $\gamma_{A}$ is the angle which describes the position of the material on the gear wheel. The stiffness at the contact position however, is dependent on which 
part of the gearwheel is in contact. The local stiffness can be obtained for an external gear by using:

$$
\begin{aligned}
& \gamma_{A}=\phi_{A}-\phi_{\text {gear }} \\
& \gamma_{B}=-\phi_{B}+\phi_{\text {gear }}
\end{aligned}
$$

Substituting Equations 25 and 26 into Equations 23 and 24 leads to the stiffness at the contact position.

$$
\begin{aligned}
& c_{\text {cont }, A}=c_{\text {const }}+c_{\Delta, A} \sin \left(2 \pi n_{\text {tooth }, A}\left(\phi_{A}-\phi_{\text {gear }}\right)\right) \\
& c_{\text {cont }, B}=c_{\text {const }}+c_{\Delta, b} \sin \left(2 \pi n_{\text {tooth }, B}\left(-\phi_{B}+\phi_{\text {gear }}\right)\right)
\end{aligned}
$$

An internal gear configuration would yield:

$$
\begin{aligned}
& \gamma_{A}=\phi_{A}-\phi_{\text {gear }} \\
& \gamma_{B}=\phi_{B}-\phi_{\text {gear }}
\end{aligned}
$$

leading to a contact stiffness of:

$$
\begin{aligned}
& c_{\text {cont }, A}=c_{\text {const }}+c_{\Delta, A} \sin \left(2 \pi n_{\text {tooth }, A}\left(\phi_{A}-\phi_{\text {gear }}\right)\right) \\
& c_{\text {cont }, B}=c_{\text {const }}+c_{\Delta, B} \sin \left(2 \pi n_{\text {tooth }, B}\left(\phi_{B}-\phi_{\text {gear }}\right)\right)
\end{aligned}
$$

The overall stiffness can be calculated by putting both springs in series:

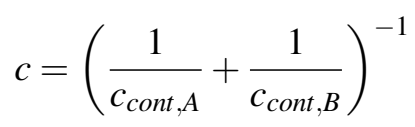

\section{Modelica Implementation}

The presented gear contact model must be supplied by constraints in the $x, y$ and $\phi$ direction (standard planar constraints). The Planar library from D. Zimmer [11] is used to supply these constraints. Features like (rotational) bearings, connection rods, inertias e.g. are all represented. The library will be used to create the total gearbox setup.

Implementation of the gear model in Modelica is straightforward using the sections above. The gear model is implemented with 2 planar interface connectors; each with 3 degrees of freedom $(x, y, \phi)$. These connectors are the connections to the gearwheels $A$ and $B$. To sense the total revolution angle $\phi_{\text {gear }}\left(\phi_{\text {gear }} \in \mathbb{R}\right)$, the atan 3 function is modified to supply a continuous and differentiable angle.

In Figure 5 the icons of the gear models are shown. No inertia's or constraints are included in the model.

Using the planar library, it is possible to create all kind of different gear configurations. Everything between

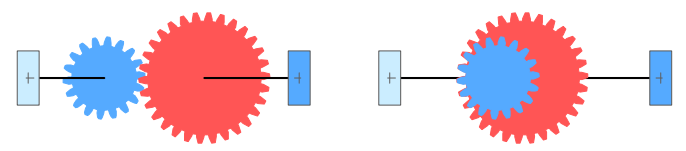

Figure 5: Modelica Icon of the inner and outer gearwheel connections

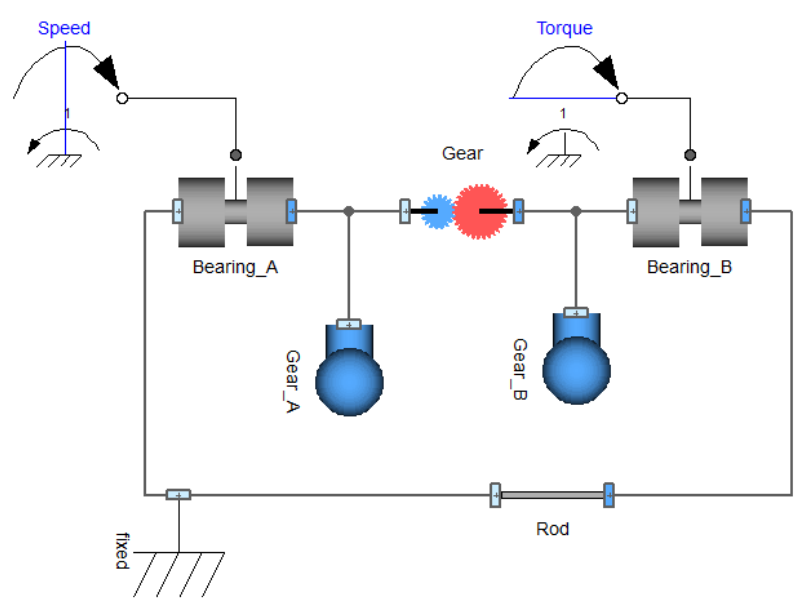

Figure 6: Spur Gear in Dymola

simple spur gears models (Figure 6 and 7) up to complex epicyclic gearing configurations (Figure 8 and 9) is easily generated. In these models, the gearbox models (Figure 5) are defined as described in this paper, all other components are components of the planar library (see [11]).

\section{Simulation Results}

\subsection{Eigenfrequency Analysis}

Using the Modelica LinearSystems2 library, it is possible to create a Bode-Diagram of a linear system. Since a linear spring and damper are used for the contact stiffness, is is possible to use this toolbox. Using an eigenfrequency analysis it is possible to check the

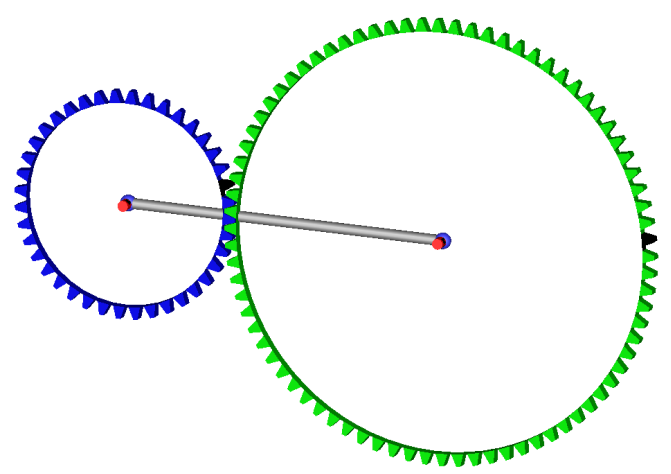

Figure 7: Spur Gear in Dymola 


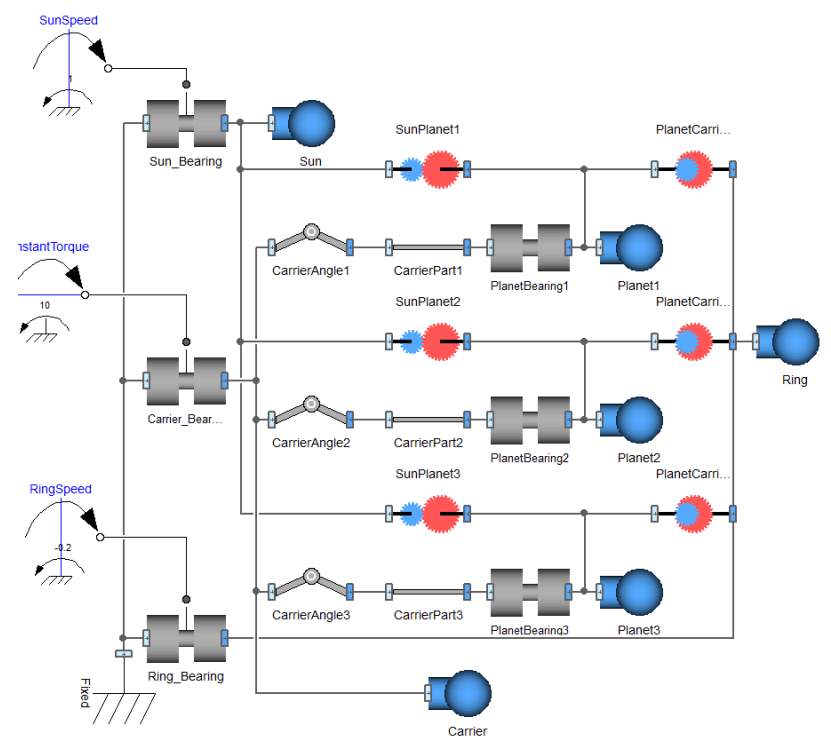

Figure 8: Epicyclic Gear behavior of the models.

\subsubsection{Spur Gear Analysis}

A Single Input Single Output (SISO) system of a simple spur gear model (as shown in Figure 6) is generated by applying a torque input on gearwheel $A$, and using as output the angular position of gearwheel $B$. The Bode-Diagram of this system ${ }^{1}$ is shown in Figure 10. In the diagram a clear peak can be found
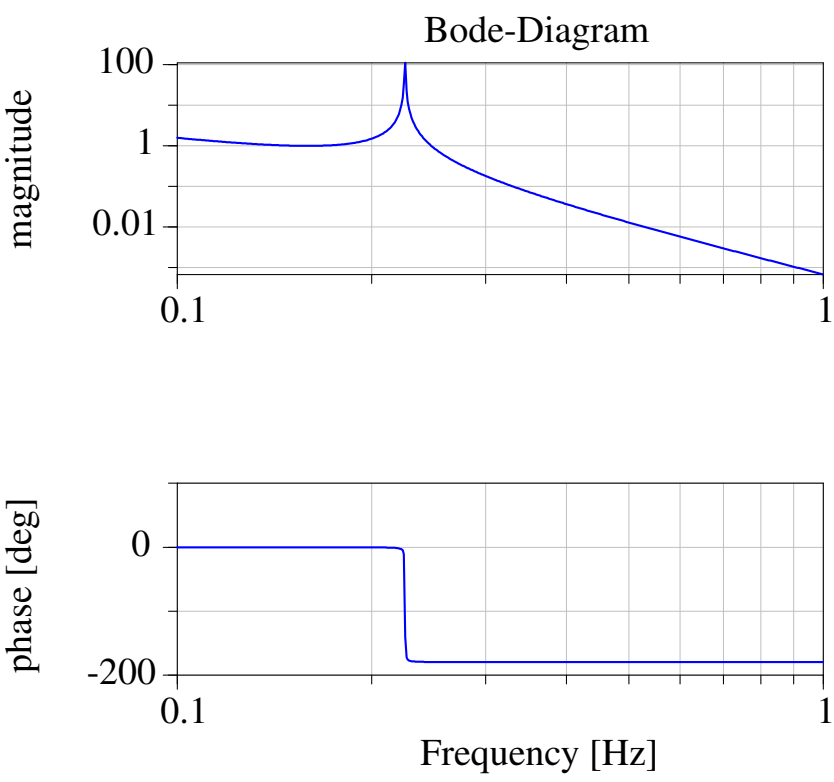

Figure 10: Bode-Diagram of the spur gear from Figure 6

at $0.225 \mathrm{~Hz}$. This is exactly the expected frequency $\omega=\frac{\sqrt{\frac{k}{m}}}{2 \pi}=\frac{\sqrt{\frac{2}{1}}}{2 \pi} \cong 0.225$. The stiffness $k=2 \frac{N}{m}$ and mass $m=1 \mathrm{~kg}$ have to be used since the system is a symmetrical system using only one spring (see e.g. [2]). Lowering of the eigenfrequency due to damping can be neglected due to the low damping coefficient.

\subsubsection{Epicyclic Gear Analysis}

A SISO system is created by defining an input torque on the sun (middle (blue) gear in Figure 9), as output the angular position of the carrier (grey structure). The Ring (red) is fixed, thereby eliminating vibrations of the ring structure. Each small planet is coupled to the planet rotating on the same axis. All bodies have the following properties: Mass $1 \mathrm{~kg}$, Inertia $1 \mathrm{kgm}^{2}$. All gear connections have a stiffness of $1 \frac{N}{m}$, and a damping coefficient of $1 \mathrm{e}-3 \frac{\mathrm{Ns}}{\mathrm{m}}$. The radius of the sun is

\footnotetext{
${ }^{1}$ The bodies have a rotational inertia of $1 \mathrm{kgm}^{2}$, the spring constant of the gear is $1 \frac{\mathrm{N}}{\mathrm{m}}$, and a damping coefficient is $1 \mathrm{e}-3 \frac{\mathrm{Ns}}{\mathrm{m}}$. Both gearwheels have a radius of $1 \mathrm{~m}$.
} 
$1 \mathrm{~m}$, the connecting planet has a radius of $0.5 \mathrm{~m}$. The other gear part of the stepped planet has a radius of $1 \mathrm{~m}$. The ring has a diameter of $2.5 \mathrm{~m}$. Using this set up, a Bode-Diagram is made (see Figure 11). When
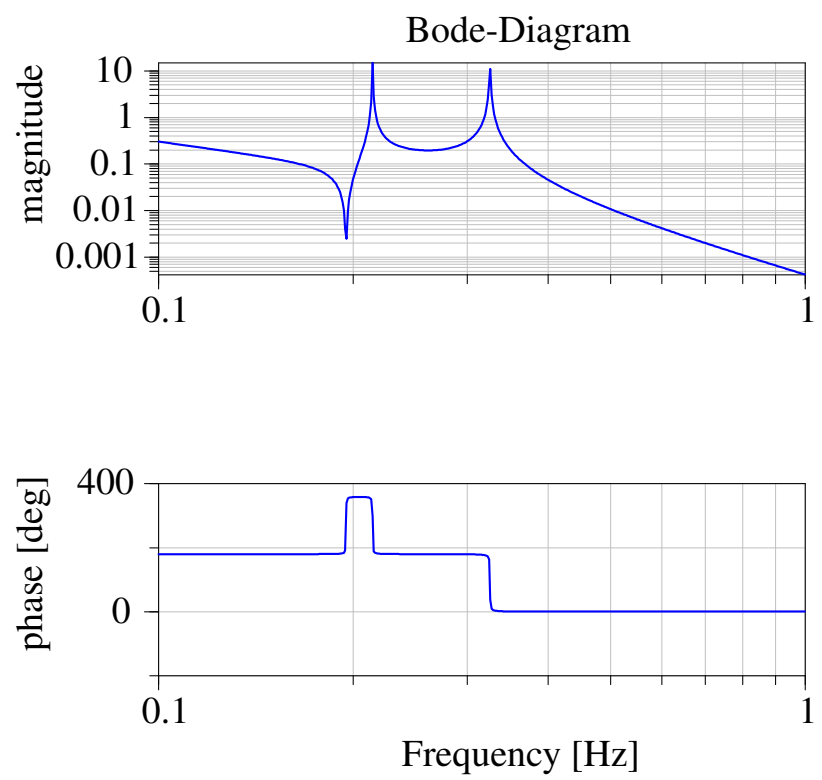

Figure 11: Bode-Diagram of the epicyclic gear from Figure 8

evaluating the Bode diagram, two peaks and a single dip can be found in the magnitude diagram. These features correspond to the 3 eigenfrequencies of the system. The fact that only 3 peaks can be found in the Bode diagram is due to the fact that the planets all have the same masses and stiffnesses. When the stiffness of one of the Sun-Planet gear connections is lowered to $0.5 \frac{N}{m}$, another peak and dip in the magnitude diagram occurs, since now one of the planets will swing in an other frequency as the others (see Figure 12).

\subsection{Internal vibrations}

In Section 4.1 the possibility of an internal excitation of the gear through varying stiffness is shown (to simulate gear mesh effects). A demonstation of this excitation is shown for a simple spur gear. Gear $A$ is accelerated from $0 \frac{\mathrm{rad}}{\mathrm{s}}$ to $1 \frac{\mathrm{rad}}{\mathrm{s}}$ with a constant acceleration. A radius of $1 \mathrm{~m}$ and 10 teeth for both gearwheels are assumed for this calculation. The constant tooth stiffness in the simulation is $1 \frac{N}{m}$, the stiffness ripple on both wheels is assumed to be $0.1 \%$. Using a damping coefficient of $0.2 \frac{\mathrm{Ns}}{\mathrm{m}}$ this yields a lightly damped system with a damping ratio $\varsigma \approx 0.071$. In Figure 13 the elastic deformation $\left(\Delta_{A B}\right)$ of the gear is shown.

In Figure 13 also shows that the system is excited by the internal mesh stiffness variation. The response of the system is the largest when the eigenfrequency of
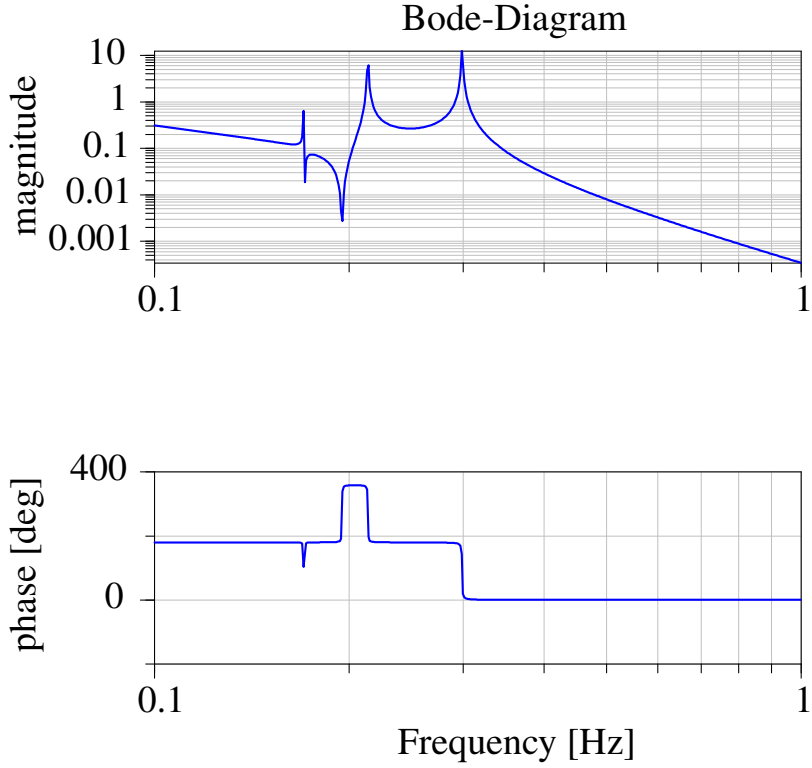

Figure 12: Bode-Diagram of the epicyclic gear from Figure 8 with reduced stiffness of one of the gear contacts.

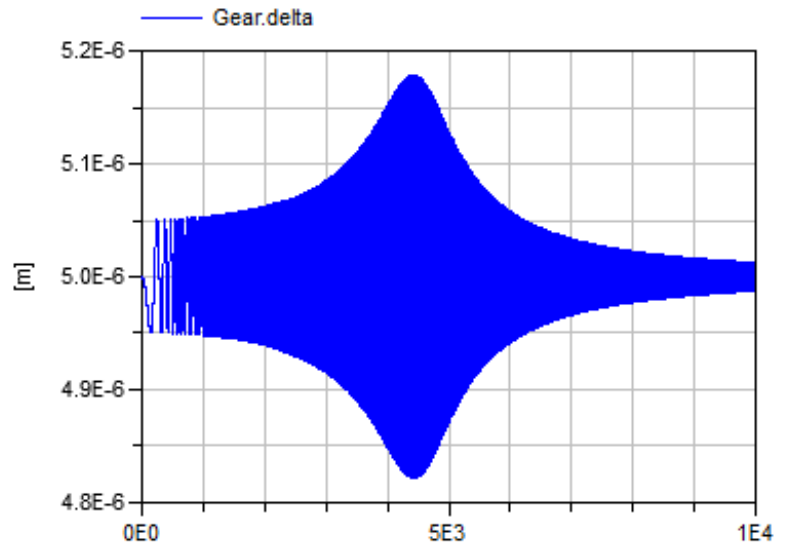

Figure 13: Time simulation of an elastic spur gear with increasing velocity.

the system approximates the excitation by the stiffness variation.

\section{Conclusion}

In this paper a model is presented to describe the contact between two gear wheels. Using an external planar library, it is possible to model arbitrary gear configurations ranging from simple spur gears up to complex epicyclic gear configurations. An option to simulate gear meshing effects by varying the stiffness of the gear contact is presented. The presented models make it possible to analyze complex gear configuration by means of time simulations as well as eigenfrequency 
analyses. The presented simulation results show the power of the method, and illustrate the capability of the model.

Acknowledgements I thank Martin Otter for his help on Modelica related issues and Dirk Zimmer for his help with Modelica and his work on the Planar library.

\section{References}

[1] Howard, I., JiA, S., AND WANG, J. The dynamic modelling of a spur gear in mesh including friction and a crack. Mechanical Systems and Signal Processing 15 (2001), 831-853.

[2] Kelly, S. Fundamentals of mechanical vibrations. McGraw-Hill series in mechanical engineering. McGraw-Hill, 2000.

[3] Ottewill, J. R., Neild, S. A., AND Wilson, R. E. Intermittent gear rattle due to interactions between forcing and manufacturing errors. Journal of Sound and Vibration 321, 3-5 (2009), 913 -935 .

[4] Özgüven, N., AND Houser, D. Mathematical models used in gear dynamics - a review. Journal of Sound and Vibration 121, 3 (1988), 383-411.

[5] Parey, A., El Badaoui, M., Guillet, F., AND TANDON, N. Dynamic modelling of spur gear pair and application of empirical mode decomposition-based statistical analysis for early detection of localized tooth defect. Journal of Sound and Vibration 294 (2006), 547-561.

[6] Parey, A., And Tandon, N. Spur gear dynamic models including defects: A review. The Shock and Vibration Digest 35 (2003), 465-478.

[7] Pedersen, R., Santos, I. F., and Hede, I. A. Advantages and drawbacks of applying periodic time-variant modal analysis to spur gear dynamics. Mechanical Systems and Signal Processing 24, 5 (2010), 1495 - 1508. Special Issue: Operational Modal Analysis.

[8] Pelchen, C., Schweiger, C., And Otter, M. Modeling and simulating the efficiency of gearboxes and of planetary gearboxes. In 2nd International Modelica Conference (2002), pp. 257-266.
[9] SAWAlhi, N., AND RANDALl, R. Simulating gear and bearing interactions in the presence of faults: Part i. the combined gear bearing dynamic model and the simulation of localised bearing faults. Mechanical Systems and Signal Processing 22, 8 (2008), 1924 - 1951.

[10] VAN DER LINDEN, F., AND VAZQUES DE SouzA SILVA, P. Modelling and simulating the efficiency and elasticity of gearboxes. In Proceedings of the 7th International Modelica Conference, Como, Italy (20-22 September 2009), pp. 270-277.

[11] ZIMMER, D. A planar mechanical library for teaching modelica. In review for the Proceedings of the 9th International Modelica Conference (2012). 
